ஆ.சு. ராஜா அ, *

அ தமிழ்த்துறை, தொன் போஸ்கோ கல்லூரி, தருமபுரி- 636809, தமிழ்நாடு, இந்தியா.

\title{
History and Culture of the Papanasa Kaani People in Anthropological Perspective
}

\author{
A.S. Raja ${ }^{a, *}$ \\ a Department of Tamil, Don Bosco College, Dharmapuri-636809, Tamil Nadu, India
}

* Corresponding Author: asraja07@gmail.com

Received: 02-06-2021 Revised: 16-01-2022

Accepted: 21-01-2022

Published: 28-02-2022

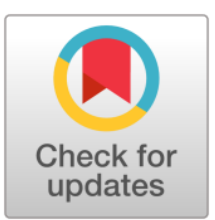

\begin{abstract}
The aim of the paper is to know the history, culture and tradition of the ancient kani people. The methodolgyis to collect the materials orally and taking personal interviews. the place is located in Tenkasi district, Ambasamudrum and pabanasam areas. In ancient days kani people used eat animals honey and Tuber and bulbs but now they eat rice and grains. They are living in huts. They are planting medicinal plants and one famous pharmacy is there. For centuries they have been giving medicine for jaundice. They are worshipping Goddess like petchiamman. They also built a church for Christian congregation.They prefer love marriages. divorce and remarriage are allowed and acceptable.
\end{abstract}

Keywords: Kanni Enathar, Aaruvikalipu, Pachilai, Molikaichedi, Enjikuli, Ellaithali

முன்னுரை

வாழ்க்கையில் மனிதன் முழு நிறைவை அடைவதற்காக மேற்கொள்ளும் முயற்சியின் பயனாக அமைவது பண்பாடு. உலகில் பல பகுதிகளில் பழங்குடியினர் வாழ்ந்து வருகின்றனர். நெல்லை மாவட்டம், அம்பாசமுத்திரம் வட்டம், பாபநாசம் மலைப்பகுதியில் வாழ்ந்து வரும் மக்களின் பண்பாட்டுக் கூறுகளை வெளிக்கொணரும் விதமாக கள ஆய்வு செய்து சேகரித்த தகவல்களைக் கொண்டும், மானிடவியல் துறை ஆய்வாதலின் களப்பணியில் அண்மையில் கண்டறிந்த தரவுகளை மனதிற்கொண்டும் இக்கட்டுரைத் தலைப்பு தேர்வு செய்யப் பெற்றது. காணி இன மக்களின் வரலாற்றின் மூலமாக மக்களின் பண்பாட்டை அறிய முடியுமா? என்பதே ஆய்வுச் சிக்கலாக அமைகிறது. காணி இன மக்களின் சமூகப் பின்புலத்தை விளக்குவதே இக்கட்டுரையின் நோக்கமாகும். காணி இன மக்களின் வரலாறு மட்டுமின்றி பழங்கால மக்களின் பண்பாட்டை அறிந்து கொள்ள முடிகிறது என்பதே இவ்வாய்வின் கருதுகோளாக அமைகிறது.

\section{இட வரையறை}

நெல்லை மாவட்டம், அம்பாசமுத்திரம் வட்டம், பாபநாசம் மலைப்பகுதி ஆய்வுக்குரிய இடவரையறையாகக் கொள்ளப்படுகிறது. களக்காட்டு மலைவரையிலும் பரந்து விரிந்து கிடக்கும் பொதியமலைப் பகுதியில் ஒரு குறிப்பிட்ட அகத்தியர் மலை எனப் போற்றப்படும் பாபநாசம் மலைப்பகுதி மேற்குத்தொடர்ச்சி மலையின் நீண்ட தொலைவையும் பரப்பையும் கொண்டது. 


\section{காணி மக்களின் வரலாறு}

பாபநாசம் மலைப்பகுதி காரையார் என்ற இடத்தில் 200 குடும்பங்கள் வசித்து வருகின்றனர். இவர்கள் அனைவரும் சிறுசிறு குடிசைகளில் வாழ்ந்து வருகின்றனர். ஆங்கிலேய ஆட்சிக்கு முன்பிருந்தே திருவனந்தபுரம் அரண்மனையில் ராஜாக்களுக்கு இடையே நடைபெறும் போரில் மலைவாழ் பழங்குடி மக்கள் அரசர்க்கு பெரிதும் உதவினர். கேரள அரசர்க்குப் போரில் உதவிய மலைமக்கள் என்பதால், உதவிய ஆதிவாசிகளுக்கு அரசன் நல்கிய மலைப் பகுதியே மேட்டுப்பகுதியாகும். நில அளவால் காணி எனப் பெயர்பெற்று இன்றும் காணி இனத்தார் என்று அழைக்கப் பெறுகின்றனர்.

“காணி நிலம் வேண்டும் - பராசக்தி

காணி நிலம் வேண்டும்"

எனப் பாரதி பாடிய பாடலில் இச்சொல் வழக்கு உள்ளது (Mahakavi Subramaniya bharathiyar, 2004). மலையாளம் தமிழ் வழங்கும் மலைப்பகுதி வாழ் ஆதிவாசிகள் காணி இனத்தார் ஆயினர்.

\section{மக்களின் பண்பாடு}

ஆங்கிலத்தில் Culture என்று குறிப்பிடுவதற்கு பண்பாடு என்ற சொல்லை முதன் முதலில் டி.கே. சிதம்பரமுதலியார் குறிப்பிடுகிறார். பெரும்பாலும் “சிறந்த வாழ்க்கைக்கு அடிப்படையாக அமைகின்ற உள்ளப்பாங்கின் வெளிப்பாட்டையே பண்பாடு என்கிறோம். உணவு, உடை மற்றும் உறைவிடம் ஆகிய மூன்றும் அடிப்படைத் தேவைகள் ஆகும். வேட்டைத் தொழிலால் கிடைக்கும் விலங்குகளின் தாடைகள் நிலத்தில் கிடைக்கும் கிழங்கு வகைகள், தேன்அடைகள் உணவுப்பொருள்களாய் ஆதியில் இ இருந்தன. தற்போது சமவெளிக்கு வந்து போவதால் தானியவகை உணவுகள் கிடைக்கிறது. மலையில் இடம் கரடு முரடாக, மேடு பள்ளமாக, இருந்தாலும் மூங்கில் கம்புகளை நட்டு குடில்கள் அமைத்துக் காய்ந்த இலை, கொடி, மரஇலைகளைப் பரப்பி வெயிலிலிருந்து காய்ந்து, தென்னை, பனை ஒலைகள் வேய்ந்து நல்ல குடிசைகள் உருவாக்கி வாழ்ந்து வருகின்றனர். நகரத்தொடர்பு ஏற்பட்டதால், பழங்காலக் குகை வாழ்வை விடுத்து, இன்று குடிசைவாழ் மக்களாய் உள்ளனர். விலங்குகளின் தொல்லைகளிலிருந்து விடுபட்டு வாழும் குழுக்களாகவே வாழ்கின்றனர். ஒவ்வொருவருக்கும் தனியாக இருப்பினும் சேர்ந்து வாழும் குடியிருப்பு பகுதியில் தான் உள்ளனர் (Dhatsanamoorthi, 2005).

குறிஞ்சி மற்றும் முல்லை நிலப் பகுதிகளைக் கொண்டது. அடர்ந்த இக்குடியிருப்புப் பகுதியில் கரடி, புலி, யானை போன்ற விலங்குகளும் முயல், மான், மிளா போன்ற அமைதியான விலங்குகளும் உள்ளன. வேட்டை நாய்களை வளர்த்து வருவதால் உணவுத் தேடலில் பாதுகாப்பும், தக்க உதவியும் இவர்களுக்கு இயல்பாக அமைந்துள்ளன. ஓயாத உழைப்பும் இவர்களிடம் இருக் கிறது. தொடக்ககால நிலையில் படிப்பறிவு அற்றவர்களாக இருந்தனர். தற்போது நாகரிகக் காலக்கட்டத்தில் அவர்களின் வாழ்க்கை முறை சற்று மாறியுள்ளது. அரசாங்கத்தின் மூலம் கிடைக்கின்ற நலத்திட்ட உதவிகளைப் பெற்றுக் கொள்கின்றனர். எளிய வாழ்க்கை முறையும், தெய்வ வழிபாடு இயற்றும் வழக்கமும் உள்ளது. பெண் தெய்வங்களில் பேச்சியம்மை வழிபாடு உள்ளது. பெண் தெய்வப் பெயர்கள் மக்கட் பெயர்களாகவும் வழங்கி வருகின்றன.

\section{மருந்து வழங்கும் பண்பாடு}

"மஞ்சள் காமாலை எனும் நோய்க்குரிய மருந்தகம் நூறாண்டுகளுக்கும் மேலாக இருந்து வரும் மலை பாபநாசம் பகுதி பொதியமலையாகும். (பொதிய மலை சிறப்பு கள ஆய்வு) மலையாளத்தைத் தாய்மொழியாகக் கொண்ட பெண் ஒருத்தியிடம் இங்கு வந்து பச்சைஇலை மருந்து பெற்றுக் குணமாகிச் சென்றோர் கணக்கில் அடங்கார். இம்மலைப் பகுதி தமிழும், நாட்டார் மருந்தும் கற்றுத்தேற வழிவகுத்ததாகும். "அகத்தியர் வாழ்ந்த மலை என்பதால் மொழிப் பண்பாடும், நோய் நீக்கத்திற்கு எளிய மருந்துகளை உருவாக்கித் தரும் மருந்து வழங்கும் பண்பாடும் மிகுந்த மலை இப்பகுதி" என்பதால் 
காணி இனமக்கள் தங்களின் நோய் நீக்கத்திற்கு மட்டுமல்லாது, பிறர்க்கும் பயன்படும் பல பச்சிலை மருந்துகளை ஆயத்தம் செய்து மக்களுக்கு உதவி வந்துள்ளனர். மருந்துச்செடி, கொடிகள் அதிகம் வளர்கின்ற மலையாக இப்பொதிய மலை விளங்குகிறது. இப்பொதிய மலையின் மேற்குப்பகுதியில் தான் திருக்குற்றால மலை அருவிகள் உள்ளன. இங்கும் அகத்தியர் அருவி உள்ளது. அருவிக்களிப்பும் ஓர் அரிய மருந்தேயாகும். மூலிகைச் செடிகொடிகளில் உராய்ந்து வரும் நீரில் நீராடினால் எவ்வித உடல்நோய் போவதுடன், உள்ளத்திற்கும் உவகை அளிக்கக் கூடியதாகும்.

சமூகத்தின் தொன்மையை ஆராயும் பொழுது பழங்குடிச் சமூகங்களை இலக்கியம், தொல்லியல் வாயிலாகவும் அறிய வேண்டியள்ளது. தமிழ்ச் சூழலில் மானிடவியல் ஆய்வுகளுக்குக் குறிப்பாக பூர்வ குடிகள் பற்றிய ஆய்வுக்கு இலக்கியம், தொல்லியல் இரண்டும் துணை செய்யும் ஆய்வுக் களங்களாகும் (Mathu.sa. Vimalantham, 2017). பழங்கால மனிதன் பிறந்த மேனியில் வாழ்ந்தான். பின் இலை தழைகழைப் பறித்து ஆடையாகக் கட்டிக்கொண்டான். விலங்குகளை வேட்டையாடிய போது தோலாடை அணிந்தான். பருத்தி விளைச்சலே அவனை நாகரிக மனிதனாக்கியது. தோலாடை அணிந்த பழங்குடியினராகிய காணியர் இன்று பருத்தி ஆடையே அணிகின்றனர். “உண்பது நாழி உடுப்பவை இரண்டு” எனும் நிலைப்பாட்டில் வாழ்ந்து வருகின்றனர் (Balasubramaniyan, 2004). இலை ஆடையின் எச்சத்தைப் பெண் தெய்வ வழிப்பாட்டில் தமிழ்நாட்டில் ஒரு கோயிலில் காணலாம். காணியர் தோலாடை அணிந்த காலம் நூறு ஆண்டுகளுக்கு முந்தைய நிலையாகும். தற்போது சமவெளி மக்கள் போலவே மகளிர் ஆடை அணிந்து வருகின்றனர். வழிபாட்டில் கிறித்துவம் இருப்பதால் தேவாலயம் ஒன்று கட்டப்பட்டுள்ளது. 137 குடும்பங்கள் வாழும் பகுதியிலும் இடப்பெயர்கள் உள்ளன. இஞ்சிக்குழி என்ற பெயர் விளையும் பயிரும், நிலஅமைப்பும் இணைந்த நிலையாகும்.

\section{முடிவுரை}

பெண் தெய்வ வழிபாட்டில் ஒரு குடிசையே கோவிலாக உள்ளது. தலைமைப்பூசாரி மறறும் துணைப்பூசாரி என இருவர் உள்ளனர். துணைப்பூசாரியே மக்களின் நோய் பற்றி அறிந்து முறையாக மந்திரம் கூறிப் போக்குகிறார். காதல் திருமணங்கள் அதிகம் நடைப்பெறுகின்றன. இலைத்தாலி அணிவிக்கப்படுகிறது. கருத்து வேறுபாடு ஏற்பட்டால் உடனே பிரிந்து மற்றொரு மணம் செய்து கொள்கின்றனர். தங்களைத் தாங்களே தனிமைப்படுத்தி வாழும் வாழ்க்கையும் இவர்களிடம் உண்டு. பழமையை மறக்காது போற்றி வாழும் மலை வாழ் மக்கள் தாம் இவர்கள் ஆயின் தற்போது மக்கள் பரந்துப் பட்ட வாழ்க்கையில் முன்னேறி வருகின்றனர். இவ்வாய்வில் ஆய்வுக்களம் விரிவாக விளக்கப்பட்டுள்ளது. தரவுகளைப் புரிந்து கொள்ள இப்பிற்புலம் பெரிதும் துணைபுரியும்.

\section{References}

Balasubramaniyan, V., (2004) Purananuru, Kashmik Printers, Chennai, Tamilnadu, India.

Dhatsana Moorthi, A., (2005) Tamilar Nagarigamum Panpadum, Yaazhl Veliyedu, Chennai, India.

Mahakavi Subramaniya Bharathiyar, C., (2004) Bharathiyar Kavithaigal, liyo Book publishers, Chennai, Tamil Nadu, India.

Mathu.sa. Vimalantham., (2017) Tamil I lakkiya Varalaru, Mullai Nilayam, Chennai, Tamil Nadu, India.

Funding: No funding was received for conducting this study.

Conflict of Interest: The Author has no conflicts of interest to declare that they are relevant to the content of this article.

About the License:

\section{(c)(1)}

Attribution 4.0 International (CC BY 4.0)
(C) The Author 2022. The text of this article is open access and licensed under a Creative Commons Attribution 4.0 International License 